\section{Lourdes et ses médecins}

\author{
A. Nussbaumer
}

la Dame. Le 23 février 1858, lors de la septième apparition, 150 personnes sont présentes, le lendemain, 300 personnes, le $1^{\text {er }}$ mars, 1500 personnes, et depuis lors le flot des pèlerins ne s'est jamais tari, actuellement plus de cinq millions par an. Lourdes est devenu l'un des sanctuaires les plus fréquentés de la chrétienté. Pour les croyants, c'est un lieu de pèlerinage, de prière, pour les non-croyants, les "miracles», non expliqués scientifiquement, sont plutôt un solde de croyances populaires aujourd'hui anachroniques.

Mes liens avec Lourdes ont motivé mon intervention auprès du Docteur Taverna. Elevé dans une famille catholique, j'ai participé aux mouvements d'étudiants et de médecins catholiques. A trente ans, médecin, en recherche, je vais en pèlerinage à Lourdes comme brancardier. Premier contact difficile, la hiérarchie à la Grotte est très stricte, le commerce florissant, mais la chaleur des malades et des brancardiers me frappe. J'y retournerai une douzaine de fois. Depuis 40 ans, dans "mon" hôpital, des brancardiers de Lourdes organisent les messes du dimanche.

En dehors de l'enceinte de la Grotte, on est frappé d'abord par un commerce florissant: images de Bernadette, de Notre-Dame, statues, chapelets, médailles, foulards, il y a de quoi satisfaire tous les goûts. L'homme aime conserver des souvenirs et ce qui touche à la religion prend un caractère sacré. On peut s'en indigner, mais aussi en sourire et respecter cette forme populaire de dévotion. dans toutes les religions, attestant la puissance du ou des Dieux, comme le miracle de "Daniel dans la fosse aux lions". Depuis l'essor scientifique du 19e siècle, les données ont changé et les miracles ont suscité de nombreuses attaques contre l'Eglise.

Le message du Docteur Taverna date de 1999. Un bref résumé historique permettra de replacer les événements dans leur contexte. Mon regard de médecin et brancardier se posera sur les pèlerins, les malades, les messages de la Dame et les miracles de demain.

\section{Bernadette Soubirous et Lourdes}

Le 11 février 1858, Bernadette Soubirous, une fille de 14 ans, pauvre, illettrée, cherche du bois le long de la rivière, le Gave, lorsqu'elle aperçoit, dans la niche d'une grande grotte, une très belle Dame habillée de blanc qui lui sourit. Ses compagnes ne voient rien. Jusqu'en juillet, la Dame lui apparaîtra encore 17 fois et lui fera des révélations. En 1862, l'Eglise catholique déclare que Marie, "l'Immaculée Conception» comme la Dame s'est appelée, est bien apparue à Bernadette. Entrée au couvent à Nevers en 1866, Bernadette, qui souffre d'asthme et de tuberculose osseuse, meurt à 35 ans, en 1879.

Bernadette n'est jamais seule devant la grotte, ceux qui l'accompagnent ne voient et n'entendent pas

Correspondance:

Dr Antoine Nussbaumer

Rue Monthéolo 16

Case postale 1226

CH-1870 Monthey 2

\section{Lourdes, terre d'Evangile}

Avant d'être au lieu de miracles, Lourdes, c'est Notre Dame, la prière, la source, les processions!

La dévotion à Marie se développe déjà chez les premiers chrétiens. L'Islam la vénère. A Lourdes, le Christ a choisi de nous envoyer sa mère, celle qui disait aux serviteurs de Cana: «Faites tout ce qu'il vous dira." Les gens de Lourdes ont vite compris que la Grotte était un lieu béni.

"Priez Dieu pour la conversion des pécheurs», telle est la première demande de la Dame à Bernadette. La seconde, quelques jours plus tard, sera d'aller «baiser la terre en pénitence pour les pécheurs", puis elle l'envoie dire aux prêtres de "venir ici en procession et d'y vive, rien d'extraordinaire, pourquoi ce rappel? Les temps sont durs, la Révolution a ébranlé la foi populaire. En 1789, la première Déclaration des Droits de l'Homme et du Citoyen a fait disparaître le Créateur.

Il m'a fallu le deuxième pèlerinage pour sentir toute la paix et la joie de Lourdes. L'essentiel ne se passe pas au niveau des corps, mais dans les cœurs. La prière, le chapelet font partie de Lourdes. Prière individuelle, à cinq heures du matin, à genoux devant la grotte, ou le soir, lorsque les cérémonies sont terminées, à la lumière des bougies qui scintillent dans la nuit. Prière communautaire aussi, lors des célébrations eucharistiques, des processions du Saint-Sacrement l'après-midi ou aux flambeaux, le soir.

L'eau fait partie du christianisme, symbole de vie et de purification, depuis le baptême jusqu'à la sébâtir une chapelle». Prier, se convertir, boire l'eau 
pulture. Jésus envoie dix lépreux se laver dans le Jourdain. La Dame prie Bernadette d'aller boire l'eau de la fontaine, qui sourd au pied de la Grotte, et de s'y laver. Depuis lors, les pèlerins boivent l'eau de la source, la ramènent chez eux pour leurs malades. Aux piscines, des bénévoles baignent dans l'eau froide malades et pèlerins qui le demandent, tout en priant le chapelet.

Pourquoi les malades viennent-ils à Lourdes? Pour être guéris? Oui, puisque c'est possible, même s'ils savent que les miracles sont rares. Il y a aussi d'autres raisons: les pèlerinages ont leur réputation, bien des paroisses les encouragent, les frais de voyage sont souvent pris en charge par de généreux donateurs. Et pourtant, certains malades pleurent, eux qui ont tant espéré! Pourquoi Dieu les laisse-t-il souffrir?

\section{Le miracle}

Le "Petit Robert» en donne la définition suivante: "fait extraordinaire où l'on croit reconnaître une intervention divine bienveillante, auquel on confère une signification spirituelle». La position de l'Eglise précise: "Dans toute guérison dite miraculeuse, il faut discerner deux niveaux: 1) le niveau scientifique médical, qui reconnaît un phénomène naturel dont la rapidité et l'ampleur sortent des règles habituelles; 2) un niveau spirituel, perçu par le bénéficiaire, qui se doit d'être respecté comme tel, puisqu'il se situe dans l'ordre de la foi.»

Depuis 2000 ans, le miracle n'a pas trouvé d'explication scientifique. Ceux qui se posent des questions devraient venir voir, ils découvriront peut-être l'invisible, cet "essentiel» dont parle le Petit Prince de Saint-Exupéry. Depuis le $19^{\mathrm{e}}$ siècle, la médecine a fait d'extraordinaires progrès. Les rationalistes de l'époque pensaient pouvoir éliminer les vieux mythes religieux. Curieuse coïncidence: à la même époque, la psychanalyse ouvre de nouveaux horizons, la psychologie analyse les structures de la personne, le corps n'est plus seul en cause, le psychisme et le spirituel prennent une place de plus en plus grande dans les causes de maladie. Mais, là aussi, rien de nouveau, si l'on se rappelle qu'Avicenne écrivait au $11^{\mathrm{e}}$ siècle les 18 volumes de son "Livre de la guérison des âmes», le Kitab al-Shifa.

Il y a cinquante ans, l'examen clinique se basait sur une observation exacte de tous les signes. Le psychisme du malade, son moral appartenaient davantage à la relation malade-médecin qu'à l'évolution de la maladie. L'Eglise, orientée vers le Christ en croix, enseignait la valeur salvatrice de la souffrance. Le temps a passé, médecins et théologiens ont appris à mieux se comprendre. Pour Jésus, guérir, c'est d'abord convertir le cœur de l'homme malade, le remettre debout, lui redonner sa vraie dignité d'enfant aimé de Dieu. La guérison miraculeuse se situe au-dessus des lois de la nature, elle ne les contredit pas, mais plutôt maîtrise les déterminismes naturels. Il n'y a pas de miracle contre nature, mais une mobilisation de toutes les forces de vie.
Les maladies guéries «miraculeusement» deviennent plus rares, aujourd'hui. On connaît, sans les expliquer, les guérisons "spontanées». Certains malades, condamnés par leurs médecins, se battent de toutes leurs forces et survivent parfois longtemps. Les médecins de Lourdes continuent à examiner toute guérison attribuée à une action divine pour dire si elle s'explique par les connaissances actuelles de la médecine ou non. Ils ont aussi droit à l'erreur, tout comme les évêques à qui il appartient de reconnaître le caractère divin de la guérison. Le miracle, lui, nous oriente nécessairement vers le Créateur, par des chemins souvent mal connus!

\section{La souffrance, un mystère}

Pourquoi Dieu accomplit-il des miracles, pourquoi guérit-il l'un et pas l'autre? Nous n'avons pas de réponse! L'Evangile nous apprend que Dieu a créé l'homme par amour en lui laissant la liberté d'accepter sa destinée. Le Christ est venu sur terre prendre notre condition, lutter contre tout mal, toute souffrance. Innocent, il a donné sa vie pour nous sauver.

Mystère de la souffrance, mystère de Dieu qui, parfois, se manifestent par un signe. Jésus dit au lépreux qu'il vient de purifier: "va te montrer au prêtre ... ta guérison sera pour les gens un témoignage». A chacun de nous de prendre position, notre liberté nous permet de croire ou de ne pas croire. Mais est-il vraiment raisonnable de nier l'existence du Créateur? 40 ans de chirurgie m'ont montré que je n'étais pas seul à vouloir guérir mes malades.

\section{Y a-t-il encore des miracles?}

Oui, il y en a encore, les deux derniers reconnus l'ont été en 1970 et 1987. Jusqu'à la fin 1999, 6784 guérisons ont été enregistrées par le Bureau médical de Lourdes et 66 reconnues miraculeuses par l'Eglise, toutes concernent des guérisons apparentes, corporelles. Les autres guérisons, celles du "cœur" ou de l'«âme" ne peuvent pas être analysées objectivement, elles sont certainement beaucoup plus nombreuses. Seuls les malades savent ce qui a pu se convertir en eux.

\section{Lourdes demain?}

La médecine moderne redécouvre les interactions entre le physique et le psychique, et la place du spirituel. L'individualisme, le rationalisme n'ont pas conduit à la paix que cherche le monde.

Lourdes offre à tous les blessés de la vie une source d'espérance, un signe de la Bonne Nouvelle!

\section{Références}

1 Taverna E. Das Wunder. Bull Med Suisses 1999;80(39):2371-2. 